\title{
Erratum to: An Experimental Framework for Generating Evolvable Chemical Systems in the Laboratory
}

\author{
David A. Baum ${ }^{1,2} \cdot$ Kalin Vetsigian ${ }^{2,3}$
}

Published online: 8 December 2016

(C) Springer Science+Business Media Dordrecht 2016

Erratum to: Orig Life Evol Biosph

DOI:10.1007/s11084-016-9526-X

Both authors are Corresponding Authors and affiliations should be shown as:

David A. Baum

dbaum@wisc.edu

Tel: 608-265-5385

Kalin Vetsigian

kalin@discovery.wisc.edu

Tel: 608-316-4670

The online version of the original article can be found under http://dx.doi.org/10.1007/s11084-016-9526-x

David A. Baum

dbaum@wisc.edu

$\triangle$ Kalin Vetsigian

kalin@discovery.wisc.edu

1 Department of Botany, University of Wisconsin, 430 Lincoln Drive, Madison, WI 53706, USA

2 Wisconsin Institute for Discovery, University of Wisconsin, 330 N. Orchard Street, Madison, WI 53715, USA

3 Department of Bacteriology, University of Wisconsin, 1550 Linden Drive, Madison, WI 53706, USA 